\title{
1 Crossing the paper chasm: Warum Digitalisierung den Point of Care erreichen muss - oder nicht stattfindet
}

\author{
Holger Cordes
}

Wir hören es und lesen es allenthalben: Das Gesundheitswesen hat ein Finanzierungsproblem. Die Alterung praktisch aller reifen Volkswirtschaften lässt die Nachfragekurve nach Gesundheitsleistungen nach rechts wandern. Der wissenschaftliche Fortschritt hat zudem den Nachteil, die „Stückkosten“ von Therapien stetig zu erhöhen, was die Angebotskurve immer steiler gestaltet. Die säkular sinkenden Wachstumsraten lassen den Geldbeutel deshalb langsamer wachsen als die Kosten. Die Lösung ist für den neoklassischen Volkswirt eine einfache Gleichung: Entweder wird das Volumen (die Versorgung) eingeschränkt, oder die Ausgaben (der Anteil am BIP) gesteigert, um in ein neues Gleichgewicht zu gelangen. Beides ist nicht attraktiv auch ohne in hässliche Details gehen zu müssen.

Für den Schumpeterianer wäre da aber noch ein dritter Weg: Innovation. Wenn also die Herausforderung im Gesundheitswesen lautet, die Stückkosten zu senken, dann bietet sich wie in anderen Branchen in erster Linie Prozesstechnologie an. Die Standardisierung von Prozessen ermöglicht immer mehr Prozesse zu digitalisieren und die Effizienz zu steigern. Damit werden zwei weitere Hebel freigesetzt: Die Standardisierung reduziert die Fehlerquote - im Krankenhaus beispielsweise die Anzahl vermeidbarer unerwünschter Ereignisse. Zudem ermöglicht die Digitalisierung eine Prozessüberwachung, die frühzeitig Trends erkennt und auf Fehlentwicklungen Gegensteuer geben kann.

Warum ergreifen Healthcare Entscheider aller Länder dann nicht die Gelegenheit beim Schopf? Warum hinkt das Gesundheitswesen weiter weit hinter der übrigen Wirtschaft hinterher? 


\section{Drei unterschätzte Ursachen}

Unabhängig von den üblichen Gründen wie Komplexität, schwaches Projektmanagement, fehlende Infrastruktur, mangelnde Budgets etc. stellt sich die Frage: Was macht die Digitalisierung im Gesundheitswesen im Allgemeinen und Krankenhäusern im Besonderen so schwierig? Aus meiner Sicht sind drei Gründe entscheidend:

1. Gesundheitsversorgung ist mobil.

2. Versorgung ist mission critical.

3. Prozesse sind ad hoc.

Der erste Grund ist hinlänglich bekannt und wird dennoch unterschätzt. Ein Flughafen ist z.B. ebenfalls eine Knowledge-Worker Organisation. Wenn Fehler gemacht werden, sind die Konsequenzen potenziell dramatisch und teuer. Die Prozesse sind ebenfalls stark fluss-gesteuert. Warum haben Flughäfen allerdings Anfang des Jahrhunderts binnen kürzester Zeit digitalisiert, während Krankenhäuser sich schwer tun? Am Flughafen arbeiten überwiegend alle Arbeitskräfte meist stationär am Computer, nur die Passagiere sind mobil. Im Krankenhaus ist das Gegenteil der Fall: Nur der Patient ist stationär, alle anderen sind immer unterwegs, Krankenschwestern sogar ca. $10 \mathrm{~km}$ jeden Tag.

Zweitens werden die Vorteile von Papier vernachlässigt: Papier hat eine 100\%ige „Uptime“. In der IT wird in „Neunen“ (99,9 ...\%) gerechnet und immer wieder übersehen, wie viel Stillstand die verbleibenden „Einsen“ bedeuten - in einem Umfeld, in dem Stillstand buchstäblich lebensbedrohlich ist. Wenn wir Papier und Telefon ablösen wollen, dann müssen wir also mission critical sein. Die Ablösung von Papier hat insofern eine metallene Komponente: „Eisen“(redundante Server, Switches, Kabel), sonst wird sie nicht funktionieren.

Die am häufigsten vernachlässigte Herausforderung für eine Digitalisierung im Krankenhaus ist allerdings der Ad-Hoc Charakter vieler Prozesse. Bis zu 50\% der Prozesse sind nicht ausreichend planbar. Selbst wenn wir die Wahrscheinlichkeit einer Komplikation in einem bestimmten Fall oder einem bestimmten Haus kennen, wissen wir nicht, wann sie wo und bei wem eintritt. Hinzu kommen die „systemischen“ Unplanbarkeiten wie Notaufnahmen, Personalfluktuation oder der Patient, der via Schwesternruf ein Glas Wasser verlangt. Ist die Hälfte aller Prozesse nicht ausreichend planbar, wirft das häufig die andere Hälfte ebenfalls über den Haufen.

Was hat das mit Digitalisierung zu tun? Das inhärente Ziel von Digitalisierung ist letztlich das Überflüssigmachen von Kommunikation. Wenn wir die richtige Information zum richtigen Zeitpunkt an den richtigen Ort bringen, in den richtigen Kontext stellen und die richtige Empfehlung geben, braucht es keine Kommunikation mehr. Was Ad-Hoc Ereignisse im Krankenhaus aber immer erfordern, ist: Kommunikation. „Ich brauche eine frische Matratze!“ oder „Wie stelle ich schnell ein Notfallteam zusammen?" Ohne Kommunikation funktioniert ein Krankenhaus nicht, selbst nicht mit dem besten Computerprogramm.

\section{Schlacht der Digitalisierung am Krankenbett}

Der preußische Stratege Carl von Clausewitz hat festgestellt, dass Schlachten nicht im Aufeinanderprallen von Massen entschieden werden, sondern an strategischen 
1 Crossing the paper chasm: Warum Digitalisierung den Point of Care erreichen muss oder nicht stattfindet

Punkten. Sieger ist, wer an diesen Punkten überlegen ist. Die Schlacht um die Digitalisierung des Gesundheitswesens wurde zu lange in den IT-Projekträumen und Rechenzentren geschlagen, obgleich der strategisch neuralgische Punkt am Patientenbett liegt.

Ein weiterer wichtiger Aspekt hat den Erfolg bisher erschwert: Die meisten der existierenden digitalen klinischen Systeme sind im Grunde für Dokumentationszwecke entwickelt worden. Insofern ist es sehr wichtig, dass sie patientenzentrisch strukturiert sind. Der Prozess am Patienten, vor allem der Pflegeprozess, ist jedoch ressourcen-zentrisch, also Pflege-, Arzt-, Transporter-, Laborassistenten-, etc. -zentrisch. Versuchen nun patientenzentrische Systeme Prozessunterstützung zu ermöglichen, kommen häufig die bekannten „Listen“ dabei heraus. Patientenzentrische Architekturen haben Schwierigkeiten, ressourcen-zentrische Prozesse abzubilden.

Zusammenfassend können wir insofern einen Zwischenstand konstatieren:

- Digitalisierung im Gesundheitswesen ist schwieriger und herausfordernder als in vielen anderen Prozessindustrien. Fügt man den oben genannten Gründen noch die Komplexität hinzu, dann bricht der Mythos vom veränderungsresistenten Gesundheitswesen in sich zusammen. Was bleibt sind die Herausforderungen.

- Digitalisierung muss am Bett anfangen und die Brücke über die „Papierschlucht“ schlagen, um in den nötigen Bereich der kritischen Masse an digitalen Daten vorzudringen. Die ist Voraussetzung, um den eigentlichen Schatz der Digitalisierung - die Prozess- und Entscheidungsunterstützung - überhaupt zu heben.

- Um den Point of Care zu digitalisieren, bedarf es Lösungen, die

- mobil und

- ausfallsicher sind,

- Kommunikationstechnologie mit Informationstechnologie verknüpfen und

n die patientenzentrische mit ressourcenzentrischen Ansätzen komplementieren.

So komplex das Umfeld der Patienten und auch der Bewohner von Pflegeeinrichtungen ist, so komplex ist darauf die Antwort. Die Digitalisierung des Points of Care muss als Integration der jeweils besten Technologie in eine bettseitige Kommunikationsplattform gedacht werden. Der Schwesternruf ist bereits am Bett, das ist ja seine originäre raison d'être. Moderne Schwesternrufsysteme können jedoch viel mehr als nur Alarme an- und abschalten. Als fest installierte Tablets können sie z.B. auch Dokumentationsworkflows übernehmen, zumal die Bett--Zuordnung ja bereits der Patienten-Zuordnung entspricht. Alarme werden heute über Middleware-Plattformen an mobile Endgeräte weitergeleitet und sind nichts anderes als hoch-priorisierte Nachrichten. Moderne Alarmeskalationssysteme sind somit auch Nachrichtenplattformen. Diese können daher genauso als Dispatcher für Tätigkeiten genutzt werden. Moderne Middlewares orchestrieren auch komplexe Alarme und die nötigen Teams, wie z.B. bei Code Blue (Reanimation). Aber auch hier kann die gleiche Technologie für weniger akute Abläufe genutzt werden und z.B. die Lieferung einer frischen Bettdecke aus dem Lager organisieren. 


\section{Vielseitige Endgeräte}

Moderne, zweckoptimierte Endgeräte bieten zudem weitere Optionen, um Abläufe datengestützt zu verbessern wie z.B. Geräte mit Infrarottechnologie, die echte Standorterkennungen bieten. Exakte Standorterkennung kombiniert mit klinischen Informationen aus der Patientenakte können somit nicht nur kontextgestützt relevante Informationen zur Verfügung stellen (z.B. Infektionswarnungen beim Eintritt in das Patientenzimmer), sondern auch die Einhaltung von Standards unterstützen (z.B. Erinnerung beim Verlassen des Zimmers, falls keine Zeit vor dem Desinfektionsspender verbracht wurde) und Reporting ermöglichen. Präzise Standorterkennung ist im klinischen Alltag meist wichtiger als eine flächendeckende Verfolgung von Ressourcen.

Als weiteres Element muss über die Anbindung und Nutzung der Daten aus bettseitigen Medizingeräten nachgedacht werden. Einerseits können Medizingeräte wie Monitore, Infusionspumpen, Beatmungsgeräte, etc. wertvolle medizinische Daten in Echtzeit liefern und digitale Entscheidungsunterstützung erst möglich machen. Andererseits erleichtert die Anbindung und digitale Verarbeitung dieser Daten viele Dokumentationspflichten und verbessert die Abrechnungsqualität. Verknüpft man jedoch die Medizingeräte mit den oben genannten anderen Elementen der bettseitigen Kommunikation (v.a. Middleware und Endgeräte), so können nicht nur Daten, sondern auch Alarme mobil und entlang der bestehenden Eskalationswege verarbeitet und Stationen geräuscharm und effizient gestaltet werden. Entscheidend ist also nicht, den Stein der Weisen zu finden, sondern die Möglichkeiten der Kommunikation und Informationsverarbeitung am Point of Care optimal miteinander und mit den peripheren Systemen wie Labor-, PDMS- oder KIS System zu vernetzen.

\section{Aktive Integration von Abläufen}

Wenn wir beginnen, Workflows nicht nur als Datenerfassung und Datenrepräsentation zu begreifen, sondern als aktive Integration von Abläufen mit der Orchestrierung von Ressourcen und Leistungserbringern, dann wird die nächste Stufe der Effizienzsteigerung im klinischen Bereich auf absehbare Zeit ohne Grenzen sein - und sich zudem in Qualitätsverbesserungen zeigen statt in fortlaufender Arbeitsverdichtung.

So wie allerdings zur Digitalisierung des Point of Care die Integration von verschiedenen Kommunikationswegen und Technologien in eine Plattform notwendig ist, so ist die spezifische Ausgestaltung dieser Plattform und die Modellierung der Abläufe in den Einrichtungen ein Ergebnis von professionellen Projekten. Zur Einführung wegweisender Kommunikations- und Digitalisierungsplattformen seien abschließend einige Ratschläge angebracht:

- Wie jedes wichtige und bewusst disruptive Projekt lebt die Digitalisierung des Points of Care von der klaren Unterstützung und aktiven Beteiligung des Managements.

- Gleichzeitig müssen Anwender von Anfang an eingebunden werden und ihren Einfluss auf die Ausgestaltung nehmen können.

- Es ist ein alter Hut, dennoch: Es gibt kein Zuviel an Kommunikation über die Ziele und das „Warum“ von Projekten oder dabei, klare Erwartungen zu setzen und Veränderungen anzukündigen. 
1 Crossing the paper chasm: Warum Digitalisierung den Point of Care erreichen muss oder nicht stattfindet

- Wandel braucht Change-Management!

- Investitionen in Infrastruktur zahlen sich immer aus. Zu viel Bandbreite gibt es nicht.

- Das Erfinden neuer Standards und Methoden ist Energieverschwendung. Nutzen Sie möglichst bewährte Konzepte.

- Lassen Sie auf keinen Fall Perfektion eine Hürde für Verbesserung werden. Heute besser ist mehr wert als morgen perfekt.

Wenn uns dies gelingt, werden wir die persistenten Informationslücken auch in mobilen und ad-hoc dominierten Prozessumgebungen am Point of Care schließen und die Versprechen der Healthcare IT einlösen können. 